\title{
Patient Safety in Surgery: All about Teamwork and Communication?
}

\author{
Lars-Peter Kamolz ${ }^{1 *}$, Stephan Spendel ${ }^{1}$, David Lumenta', Gerald Sendlhofer ${ }^{1,2}$ \\ From Safety in hospitals: from strategy to implementation Annual Scientific Meeting 2015 \\ Graz, Austria. 29-30 September 2015
}

\section{Background}

Patient safety is an essential topic for hospitals by preventing the occurrence of adverse events with the support of properly implemented security systems [1]. Promoting patient safety is a fundamental part of a systems approach in order to create a certain safety culture, especially for surgical departments.

Errors in surgical specialties, as compared with other settings, can be potentially devastating and may lead to high-profile effects for surgeons and their institution. Adequately installed communication in a safety-enhancing environment can prevent such events. A study by the Joint Commission on Accreditation of Health-care Organizations (JCAHO) identified communication as the root cause for sentinel events in surgery $[1,2]$. Therefore, we posed the following question "Are we really safe?"

\section{Material and methods}

Donabedian's model of quality and thereby safety was categorizes measures in structure, process, and outcome. We used operating room briefings and debriefings as well as video-based adult learning on how to best use a surgical safety checklist (SSC). The video supports to better understand the correct use of a SSC as well as to temper sentinel events by promoting teamwork facilitated by improved communication. At the beginning of the Team-Time-Out employees introduce themselves. Further items are asked during the Team-Time-Out as suggested by the World Health Organization. We also conducted a Sign-out at the end of the surgery in order evaluate any improvements for the future.

\section{Results}

Using the surgical safety checklist is associated with an improved patient safety and enhances safety culture. Thereby it also facilitates to save costs due to optimized processes and lessons learned.

\section{Conclusions}

Briefings and debriefings helped to minimize errors by allowing employees to discuss potential problems before leading to a "near miss" or actual harm. Nevertheless, further steps are needed to increase communication and team-approach in surgical disciplines [1].

\section{Authors' details}

${ }^{1}$ Division of Plastic, Aesthetic and Reconstructive Surgery, Department of Surgery, Medical University of Graz, Graz, Austria. ${ }^{2}$ Department of Quality and Risk Management, University Hospital Graz, Graz, Austria.

Published: 30 October 2015

\section{References}

1. Makary MA, Sexton JB, Freischlag JA, Millman EA, Pryor D, Holzmueller C, Pronovost P: Patient safety in surgery. Ann Surg 2006, 243(5):628-635.

2. Joint Commission on Accreditation of Healthcare Organizations: Sentinel Events: Evaluating Cause and Planning Improvement Oakbrook Terrace, IL: Joint Commission on Accreditation of Healthcare Organizations; 1998.

doi:10.1186/2056-5917-1-S1-A20

Cite this article as: Kamolz et al:: Patient Safety in Surgery: All about Teamwork and Communication? Safety in Health 2015 1(Suppl 1):A20.

\footnotetext{
* Correspondence: lars.kamolz@medunigraz.at

'Division of Plastic, Aesthetic and Reconstructive Surgery, Department of

Surgery, Medical University of Graz, Graz, Austria

Full list of author information is available at the end of the article
} 